\title{
Anti-TIGIT Monoclonal Antibody AB154
}

National Cancer Institute

\section{Source}

National Cancer Institute. Anti-TIGIT Monoclonal Antibody AB154. NCI Thesaurus. Code C162875.

A humanized immunog lobulin G1 (IgG1) monoclonal antibody targeting the co-inhibitory molecule and immune checkpoint inhibitor T-cell immunoreceptor with immunog lobulin (Ig) and immunoreceptor tyrosine-based inhibitory motif (ITIM) domains (TIGIT), with potential immune checkpoint inhibitory activity. Upon administration, anti-TIGIT monoclonal antibody AB154 targets and binds to TIGIT expressed on various immune cells, particularly on tumor-infiltrating T-lymphocytes (TILS), thereby preventing the interaction of TIGIT with its ligands CD112 (nectin-2; poliovirus receptor related-2; PRR2; PVRL2) and CD155 (poliovirus receptor; PVR; nectin-like protein 5; NECL-5). This enhances the interaction of CD112 and CD155 with the costimulatory receptor CD226 (DNAX Accessory molecule-1; DNAM-1), which is expressed on immune cells, such as natural killer (NK) cells and CD8+ T-cells. This leads to CD226 dimerization and CD226mediated signaling and activates the immune system to exert a T-cell-mediated immune response against cancer cells. TIGIT, a member of the Ig super family (IgSF) and an immune inhibitory receptor, plays a key role in the suppression of T-cell proliferation and activation; it is involved in tumor cell immune evasion, and the inhibition of antiviral immune responses. 